\title{
Role of sphingolipids in senescence: implication in aging and age-related diseases
}

\author{
Magali Trayssac, ${ }^{1}$ Yusuf A. Hannun, ${ }^{1}$ and Lina M. Obeid ${ }^{1,2}$ \\ ${ }^{1}$ Stony Brook Cancer Center and Department of Medicine, Stony Brook University, Stony Brook, New York, USA. ${ }^{2}$ Northport Veterans Affairs Medical Center, Northport, New York, USA.
}

\begin{abstract}
Aging is defined as the progressive deterioration of physiological function with age. Incidence of many pathologies increases with age, including neurological and cardiovascular diseases and cancer. Aging tissues become less adaptable and renewable, and cells undergo senescence, a process by which they "irreversibly" stop dividing. Senescence has been shown to serve as a tumor suppression mechanism with clear desirable effects. However, senescence also has deleterious consequences, especially for cardiovascular, metabolic, and immune systems. Sphingolipids are a major class of lipids that regulate cell biology, owing to their structural and bioactive properties and diversity. Their involvement in the regulation of aging and senescence has been demonstrated and studied in multiple organisms and cell types, especially that of ceramide and sphingosine-1-phosphate; ceramide induces cellular senescence and sphingosine-1-phosphate delays it. These discoveries could be very useful in the future to understand aging mechanisms and improve therapeutic interventions.
\end{abstract}

\section{Introduction}

Sphingolipids, a major class of lipids present in all eukaryotic cells, are essential to organismal development and maintenance (1). Thudichum first isolated sphingolipids from brain tissue in 1884, dubbing them "sphingosins" because of their enigmatic nature. This group of lipids includes over 300 members that have a common sphingoid base called sphingosine (Sph), an amino-alcohol backbone most often composed of 18 carbons that form the nonpolar tail. The sphingoid base can be bound to a fatty acid via an amide link to produce ceramide (Cer). Two main types of more complex sphingolipids are distinguished by their polar head group: in glycosphingolipids, the polar head is based on a glycosidic group, and in sphingophospholipids, the polar head contains a phosphate group (2).

Sphingolipids are bioactive molecules that regulate cell biology and fate such as cell cycle, senescence, proliferation, and migration (3). The bioactive sphingolipids include Cer, Sph, sphingosine-1-phosphate (S1P), ceramide-1-phosphate (C1P), and others. Their roles have proven crucial in many functions, such as immune responses, inflammation, cancer, metabolic and cardiovascular diseases, and neurodegeneration (4).

During aging, organisms progressively lose physiological functions (5). Aging is a complex phenomenon that is not strictly dependent on chronological age, but some pathways and mechanisms are conserved over time and species (6). Cellular senescence is known to contribute to aging and usually forms the basis for biological studies because of its intimate connections with tissue aging; moreover, many senescence-associated inducers, effectors, and markers have been identified over the past 50 years (7). Senescence corresponds to a pseudopermanent proliferative

Conflict of interest: The authors have declared that no conflict of interest exists. Reference information: J Clin Invest. 2018;128(7):2702-2712.

https://doi.org/10.1172/JCI97949. arrest (as it can be reversed under some conditions) in cells in response to diverse stimuli. Since its first description by Hayflick and Moorhead in 1961 in primary fibroblasts (8), senescence has been observed not only in cells but in tissues of different organisms, making it a pivotal event in the aging process (9). As such, senescence likely plays a large role in the age-related risk for prevalent human diseases including cancer, neurodegeneration, cardiovascular dysfunction, and type 2 diabetes (10).

In this Review, we focus on describing links between sphingolipids and aging and age-related diseases. From yeast to clinical data in centenarians, we will discuss new and exciting findings that could have significant impact.

\section{Overview of sphingolipid metabolism}

Complexity of sphingolipid metabolic pathways. Sphingolipid metabolism is a complex, interconnected network of molecules. Cer, playing the role of a central metabolic hub (11), can be synthesized by multiple pathways, including three main pathways: the de novo pathway, hydrolysis of complex sphingolipids, and the salvage pathway (Figure 1 and ref. 12).

The de novo pathway occurs at the ER membrane and is characterized by the condensation of serine and palmitoyl-CoA through the action of serine palmitoyltransferase (SPT) (13). SPT is a protein complex of three subunits called SPTLC1, SPTLC2, and SPTLC3 $(14,15)$. The 3-ketodihydrosphingosine formed is reduced to dihydrosphingosine ( $\mathrm{dhSph}$ ), which is then acetylated by (dihydro)ceramide synthases (CerSs) to dihydroceramide (dhCer). Six CerS isoforms are identified in mammals, and each has a preference for different fatty acyl chain lengths (16). For instance, CerS1 synthesizes C18dhCer, CerS2 synthesizes verylong-chain Cer (C22, C24, and C26dhCer), and CerS5 and CerS6 catalyze the formation of $\mathrm{C} 14$ and $\mathrm{C} 16 \mathrm{dhCer}$. The desaturases (Des) then desaturate dhCer to generate the corresponding Cer. 


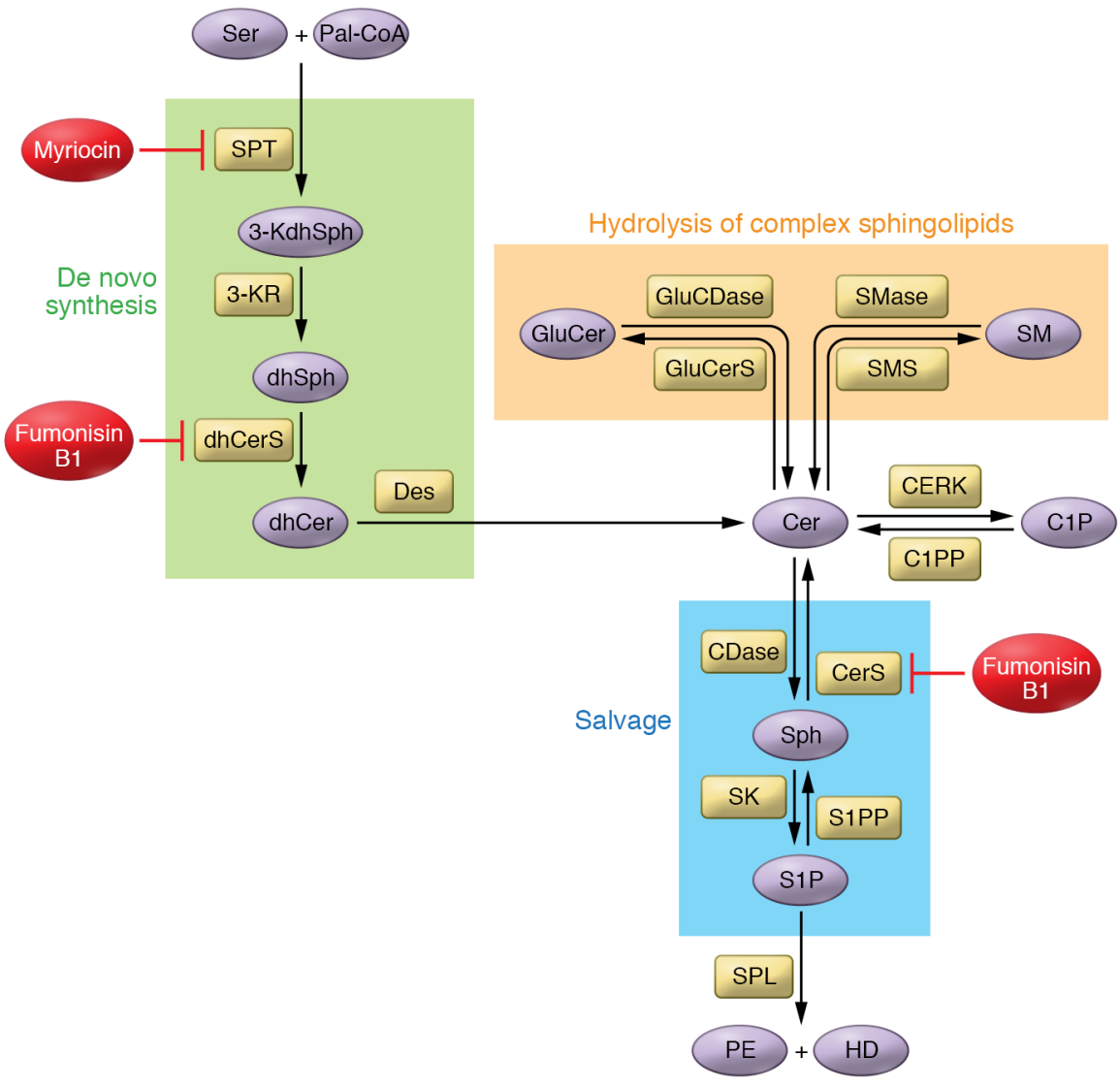

Figure 1. Overview of sphingolipid metabolism. Cer plays a central role in the complex network of sphingolipid metabolism and can be synthesized by many different enzymes belonging to different pathways. Cer is also one of the most potent bioactive sphingolipids. It can regulate cell biology by inducing cell cycle arrest and senescence. Its role in cell death has been extensively investigated. The few direct targets of Cer that have been discovered are mostly protein phosphatases. By activating them, Cer can modulate signaling pathways. Another very well-studied sphingolipid is S1P, which has been shown to induce cell proliferation and migration among other functions. Its actions are mostly known through its ability to activate GPCRs, leading to modifications of cell biology. Two pharmacological inhibitors have been extensively used to study sphingolipid metabolism: myriocin inhibits sphingolipid synthesis through the inhibition of SPT, and fumonisin B1 is a CerS inhibitor, inhibiting de novo and salvage pathways. Des, dihydroceramide desaturase; dhCerS, dihydroceramide synthase; GluCDase, glucosylceramidase; GluCerS, glucosylceramide synthase; HD, hexadecenal; 3-KdhSph, 3-ketosphingosine; 3-KR, 3-ketoreductase; Pal-CoA palmitoyl-CoA; PE, phosphoethanolamine; S1PP, S1P phosphatase.
Hydrolysis of complex sphingolipids. Sphingomyelin (SM), the most abundant sphingolipid in mammalian cells, can be degraded into Cer and phosphocholine by sphingomyelinases (SMases). There are three families of SMase - acid (aSMase), neutral (nSMase), and alkaline - based on their optimal pH of action (17). Conversely, sphingomyelin synthases (SMSs) use phosphatidylcholine and Cer to form SM and diacylglycerol (DAG) (18). Two SMSs have been identified, and they regulate complex biologies as they utilize Cer to produce DAG, an important bioactive glycerolipid that often exerts effects on cell growth and survival opposite those of Cer.

Degradation of glycosphingolipids can be another source of Cer through the action of many hydrolases, culminating in the action of glucosidase and galactosidase on glucosylceramide (GluCer) and galactosylceramide (GalCer), respectively.

In the salvage pathway, also called the recycling pathway, multiple hydrolytic enzymes, including SMases, glucosidase, and ceramidases (CDases) (19), produce Sph, which can be reacylated to Cer by CerS. (Note that CerS can utilize Sph to directly produce Cer; ref. 20.) CDases mediate the hydrolysis of Cer, producing Sph. Like SMases, CDases constitute a family of enzymes categorized by preferred pH: acid (aCDase), neutral (nCDase), and alkaline (21).

Sphingosine kinases (SKs) phosphorylate Sph to form S1P. Two SK isoforms have been identified: SK1 and SK2. While SK1 is located in the cytosol and at the plasma membrane, SK2 is mostly present in the nucleus and ER (22). The product S1P acts as a second messenger on intracellular targets (23) and as primary ligand on extracellular receptors (24). S1P can be converted back to Sph through the action of S1P phosphatases (SPPs). On the other hand, the S1P lyase (SPL) irreversibly degrades S1P into phosphoethanolamine and hexadecenal. This unique reaction provides the only known exit to sphingolipid metabolism.

Similarly, ceramide kinase (CERK) phosphorylates Cer into C1P, and C1P phosphatase (C1PP) dephosphorylates C1P to regenerate Cer.

Central roles of Cer and S1P in cell biology. While Cer and Sph are usually involved in induction of cell cycle arrest, senescence, apoptosis, and cell differentiation, S1P promotes cell survival, proliferation, migration, invasion, and angiogenesis (25). S1P is also implicated in immune responses and inflammation. As a consequence, enzymes involved in producing and degrading Cer, Sph, and S1P are crucial regulators of sphingolipid bioactivity.

S1P acts primarily through extracellular receptors (26), although it has been shown to have several intracellular targets, such as histone deacetylase (23), prohibitin 2 (27), and human telomerase reverse transcriptase (hTERT) (28). S1P acts on five GPCRs, the S1P receptors (S1PRs), following its extracellular export by ATP-binding cassette transporters (29) and the key transporters Spinster 2 (30) and major facilitator superfamily domain-containing 2b (Mfsd2b) in red blood cells (31). S1P binding to S1PR triggers canonical GPCR signaling (32) such as Rac, ERK, PI3K/Akt, PLC, and Rho pathways (33). Many SK inhibitors based on analogs of Sph have been used, but they generally lack specificity and potency (micromolar concentrations) (34). FTY720, also called fingolimod, is an Sph analog that is phosphorylated by SK2 and then acts as an S1PR agonist, leading to receptor internalization and downregulation, functionally suppressing S1P 


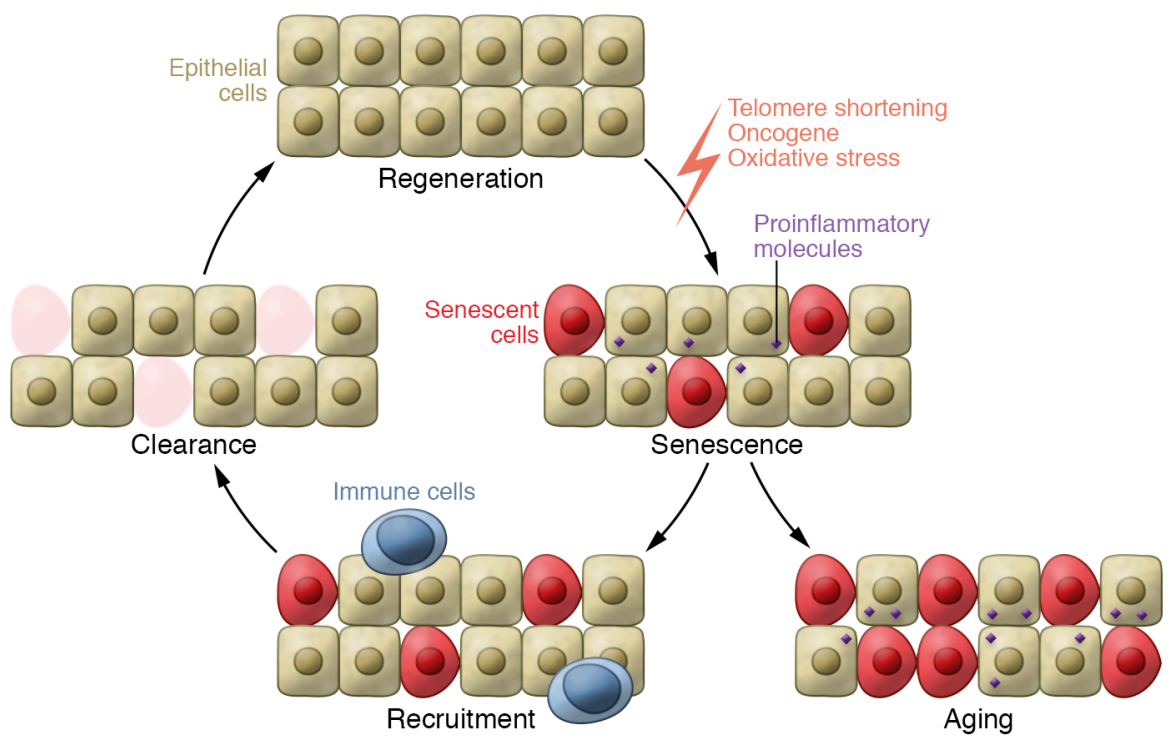

Figure 2. Senescence and consequences for tissue homeostasis. Senescence can have beneficial effects when acute because it allows the tissue to adapt to stress. Senescent cells secrete proinflammatory molecules in order to recruit immune cells that can then lead to clearance of senescent cells in the damaged tissue. If the regenerative capacity is efficient, tissue homeostasis is maintained. Senescence is a tumor suppressor mechanism and has some beneficial effects during wound healing and fibrosis. Aging induces a decrease in the efficiency of this cycle of senescence/recruitment/clearance/regeneration. Senescence can have deleterious effects when chronic, worsening pathologies such as type 2 diabetes and atherosclerosis. signaling (35). FTY720 is used clinically for treatment of multiple sclerosis through suppressing the immune system by preventing lymphocyte egress from lymphoid tissues (36). Other nonlipid compounds, such as PF-543 (37), SKI-II (38), and ABC294640 (39), are high-potency SK inhibitors in vitro.

Cer activates serine/threonine protein phosphatases such as PP1 and PP2A (40). For example, PP2A activation leads to dephosphorylation and inactivation of Akt and retinoblastoma protein (pRb) (41). Cer is hydrophobic, and therefore its delivery to cells is limited. However, it can flip readily across membrane leaflets and shuttle between distinct cellular membranes through two mechanisms: via vesicular transport or via transfer proteins. Indeed, Cer trafficking from ER to Golgi apparatus is partly mediated through Cer transfer protein (CERT), which selectively couples Cer with SM synthesis (42). Cer can be delivered to cells primarily in shortchain forms such as C2-, C6-, and C8-Cer, or using nanoliposomes (43), whereas longer-chain Cer can be delivered by dodecane/ ethanol (44). Inhibitors can modulate the activity of sphingolipid enzymes involved in Cer synthesis. For example, fumonisin B1 (FB1) is a CerS inhibitor that inhibits Cer formation through both de novo and salvage pathways, whereas myriocin (Myr), an SPT inhibitor, inhibits the de novo pathway at its root (Figure 1).

\section{Overview of senescence}

Senescence is a mechanism by which cells adapt to environmental stress. It leads to tissue adaptation after diverse injuries, but it can also result in poorly functioning cells and tissues. Senescence is a multistep process (Figure 2): cells stop dividing, and tissues recruit immune cells that lead to clearance of senescent cells and tissue regeneration $(45,46)$. The current hypothesis is that acute senescence is protective whereas chronic senescence can be harmful for tissue homeostasis (47). As a result, pro-senescence therapies could be beneficial for early-stage diseases, but anti-senescence strategies might be of interest to prevent chronic tissue damage occurring in late-stage diseases (48).

Inducers and effectors of senescence. Some well-characterized senescence inducers include telomere shortening; DNA damage-inducing agents such as ultraviolet or gamma irradiation, chemotherapeutics, oncogene activation, or tumor suppressor inactivation; and ROS (49).

Telomeres become progressively shorter after each cell division, leading to activation of the DNA damage response (DDR) (50). Ataxia-telangiectasia-mutated protein (ATM) is recruited to the telomeres and induces 53 phosphorylation and stabilization (51). Activated p53 then induces p 21 transcription, which inhibits cyclin-dependent kinase 2 (CDK2) and leads to pRb hypophosphorylation. In this state, active $\mathrm{pRb}$ sequesters the transcription factor E2F, preventing the expression of genes required for DNA synthesis that occurs during the $\mathrm{S}$ phase of the cell cycle (52).

Aging induces a derepression of the CDKN2A tumor suppressor locus (53), which codes for two distinct proteins: p16 and ARF (also called p19 in mice and p14 in humans). p16 inhibits CDK4 and CDK6 and inhibits the transition from $G_{1}$ to $S$ phase. ARF increases the stability of $\mathrm{p} 53$ protein and consequently increases p21 expression (54). p21 upregulation activates the p16 pathway, which is involved in maintaining the senescent state, whereas the p21 pathway is the mechanism inducing senescence (55). Cooperation between these two pathways varies depending on the context.

Oncogenic activation, especially proteins from the Ras family, BRAF, and c-myc, induces senescence in vitro and in vivo. Reciprocally, the loss of PTEN or VHL, well-known tumor suppressors, can induce senescence (56). Oncogene-induced senescence can be DDR-dependent (57) or -independent, mediated through the p16 and ARF pathways.

p21 can induce ROS accumulation that is crucial for the induction of senescence (58). p38 MAPK has also been shown to be an important mediator of oxidative stress-induced senescence (59).

Markers of senescence. Senescent cells exhibit some specific characteristics other than cell cycle arrest. They have increased activity of the lysosomal hydrolase $\beta$-galactosidase, also called senescence-associated $\beta$-galactosidase (SA $\beta$-gal) activity, likely the consequence of an expanded lysosomal compartment during cell senescence. SA $\beta$-gal is widely used to detect senescent cells by incubating them with the substrate of the enzyme X-gal at $\mathrm{pH} 6$ and measuring the formation of the product, an insoluble blue pigment (60). 


\section{Table 1. Summary of the role of sphingolipids in regulating lifespan}

\begin{tabular}{|c|c|}
\hline Organism & Role of sphingolipids in lifespan \\
\hline \multirow[t]{3}{*}{ Saccharomyces cerevisiae } & Deletion of a gene coding for CerS (longevity-assurance gene 1, LAG1) results in an increase of mean and maximum lifespan (134). \\
\hline & $\begin{array}{l}\text { Downregulation of sphingolipid synthesis induces an increase of lifespan, shown by lowering the expression of the SPT subunits LCB1 and LCB2, or by } \\
\text { using the SPT pharmacological inhibitor Myr (135). }\end{array}$ \\
\hline & $\begin{array}{l}\text { During aging, there is an increase of sphingoid bases (136). It is thought to be the result of the upregulation of alkaline CDase (YPC1 and YDC1) and the } \\
\text { downregulation of the sphingoid base kinases LCB4/5. Age-induced increase in sphingoid bases was shown to impair mitochondrial structure and functio } \\
\text { and to decrease lifespan. }\end{array}$ \\
\hline \multirow[t]{3}{*}{ Caenorhabditis elegans } & $\begin{array}{l}\text { Cer and SM accumulate during aging. Targeting SPT by siRNA or with Myr was also shown to extend mean and maximum lifespan in the worm, whereas } \\
\text { animals fed a diet rich in sphingolipids have a reduced lifespan (137). }\end{array}$ \\
\hline & $\begin{array}{l}\text { Two CerSs, HYL-1 and HYL-2, seem to have opposite effects on aging. Loss of HYL-1 was shown to have no strong effect on lifespan according to one study } \\
\text { (138), but another study showed decreases in some signs of aging (139). In contrast, loss of HYL-2 decreases lifespan (138). The disparity is possibly due } \\
\text { to differing roles in the synthesis of distinct Cers, such that very-long-chain Cer induces aging whereas shorter ones delay it. }\end{array}$ \\
\hline & SK1-deficient animals have a shorter lifespan (139). \\
\hline \multirow[t]{2}{*}{ Drosophila melanogaster } & Inactivation of Dacer, the Drosophila alkaline CDase, induces Cer accumulation and increases lifespan and antioxidative stress capacity of the flies (140). \\
\hline & $\begin{array}{l}\text { Flies lacking a functional CERT have an important decrease of SM and Cer, which correlates with increased membrane fluidity and susceptibility to ROS, } \\
\text { resulting in premature aging and death (141). }\end{array}$ \\
\hline
\end{tabular}

Senescent cells also lose lamin B1 expression (61). This downregulation leads to deep changes in chromatin organization and transcriptional modifications. Indeed, senescence-associated heterochromatin foci are characteristic of senescent cell nuclei.

The senescence-associated secretory phenotype (SASP) is a widely studied cellular program and marker of senescence (62). Senescent cells express and secrete a wide range of molecules, from cytokines and chemokines to growth factors and matrix metalloproteinases (MMPs), including IL-6, IL-1, IL-8, MCP-2, GM-CSF, bFGF, TGF- $\beta$, and others. SASP induction is dependent on the DDR, but p38 signaling also seems to play a role. Both pathways activate $\mathrm{NF}-\kappa \mathrm{B}$, the main signaling pathway that triggers SASP (63).

\section{Role of sphingolipids in senescence and aging}

Studies in yeast, worms, and flies have shown that sphingolipids play critical roles in regulating lifespan. Indeed these studies, using genetic and biochemical approaches, demonstrate conclusively the involvement of sphingolipids in aging of these model organisms (summarized in Table 1). In mammalian cells, evidence has been accruing on roles of sphingolipids in the regulation of senescence (Figure 3). These roles are discussed here because they are more relevant to human pathobiology.
Over two decades ago, our group showed increased Cer levels in "old" senescent ( $>55$ passages) WI-38 fetal-lung-derived human fibroblasts compared with "young" ones (<30 passages) (64). nSMase activity was much higher in senescent fibroblasts, whereas aSMase activity was only slightly increased (64). Mechanistic results demonstrated Cer's ability to induce a senescent phenotype whereby C6-Cer inhibited growth and DNA synthesis in young fibroblasts in the 5 -to- $15-\mu \mathrm{M}$ range, but was cytotoxic when used at $20 \mu \mathrm{M}$ and more. C6-Cer also induced a complete

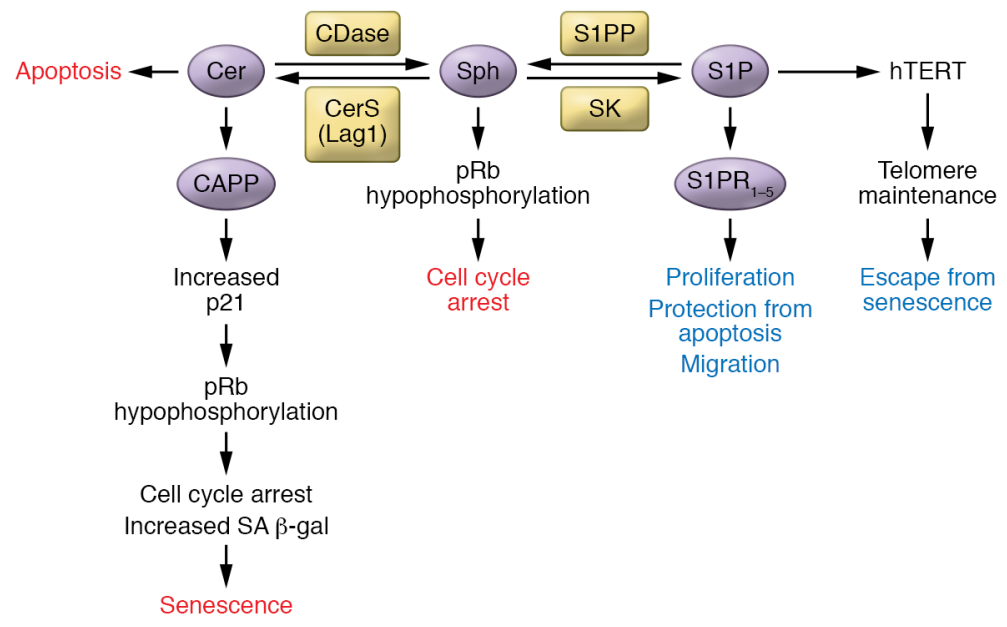

Figure 3. Mechanisms by which sphingolipids regulate senescence. Cer and S1P have opposite effects on senescence: while Cer induces it, S1P seems to prevent it. The mechanisms by which Cer and S1P regulate senescence are very distinct. Studies on the mechanisms induced by Cer are numerous, whereas little is known about the mechanisms activated by S1P to modulate senescence. CAPP, ceramide-activated protein phosphatase. 


\section{Table 2. Summary of the role of senescence and sphingolipids in regulating tissue functions}

\begin{tabular}{|c|c|c|}
\hline Pathology & Role of senescence & Role of sphingolipids \\
\hline Cancer & Beneficial and deleterious & $\begin{array}{l}\text { Cer mediates oncogene-induced senescence and potentiates the effects of chemotherapeutics } \\
\text { In p53-deficient tumors, loss of SK1 induces senescence and inhibits tumor development } \\
\text { SK2-derived S1P delays senescence and promotes tumor growth } \\
\text { Senescence can be protumorigenic through SASP, but the role of sphingolipids in this context is unknown }\end{array}$ \\
\hline Fibrosis & Beneficial & Cer induces senescence, which has antifibrotic effects and might help tissue recovery \\
\hline Type 2 diabetes & Deleterious & Cer induces senescence in adipocytes, hepatocytes, and myoblasts, leading to insulin resistance \\
\hline Immunosenescence & Deleterious & $\begin{array}{l}\text { Cer decreases proliferative capacity of T cells } \\
\text { Cer mimics effect of aging in macrophages }\end{array}$ \\
\hline Alzheimer's disease & Deleterious & $\begin{array}{l}\text { Cer promotes the biogenesis of amyloid } \beta \text {-peptide } \\
\text { Cer-enriched exosomes exacerbate the disease } \\
\text { Inhibition of aSMase or nSMase } 2 \text { protects from the disease }\end{array}$ \\
\hline
\end{tabular}

dephosphorylation of $\mathrm{pRb}$ in young fibroblasts, mimicking observations in senescent fibroblasts. In a different human fibroblast line, IMR-90, Cer levels were observed to be twofold higher in senescent fibroblasts compared with young fibroblasts, whereas the level of Sph was approximately $50 \%$ lower (65).

In human leukemic cell lines such as MOLT- 4 and in murine fibroblasts (L-929), C6-Cer induced cell cycle arrest in $G_{0} / G_{1}$ phase but did not upregulate p53. C6-Cer-induced cell cycle arrest was mediated by retinoblastoma protein $(\mathrm{Rb})$ hypophosphorylation and was p53-independent, implying that p53 is not a downstream target of Cer and is not necessary for Cer's growth-suppressive effects (66). Moreover, in WI-38 fibroblasts, $\mathrm{G}_{1}$ arrest induced by serum deprivation did not increase Cer levels, whereas release of nocodazole-induced $\mathrm{G}_{2} / \mathrm{M}$ arrest led to an increase in Cer, reversed by FB1, implicating a role for CerS in the Cer increase. FB1 also inhibited pRb dephosphorylation that occurred during the progression from $G_{2} / M$ to $G_{1}$. Thus, de novo synthesized Cer modulates $\mathrm{Rb}$ phosphorylation and cell cycle progression (67).

C6-Cer has also been shown to induce SA $\beta$-gal expression (68). In WI-38 cells, induction of $\beta$-gal expression by C6-Cer was time- and concentration-dependent and correlated with inhibition of DNA synthesis. Incubating the cells for 15 days with $5 \mu \mathrm{M}$ C6-Cer induced $\beta$-gal expression in $90 \%$ of the cell population.

Further investigation revealed that C6-Cer directly activates PP1 and PP2A, which then inhibit CDK2, leading to cell cycle arrest. C6-Cer also induced increased p21 expression and association with CDK2 but did not affect CDK4, p16, or p27 (69).

Other sphingolipids have been investigated in the regulation of cell cycle and senescence. In MOLT- 4 cells, Sph induced cell cycle arrest in $G_{0} / G_{1}$ and $p R b$ dephosphorylation in a dose- and time-dependent manner, from 100 to $500 \mathrm{nM}$. The mechanisms are unknown but probably occur through the activation of a phosphatase or inhibition of a kinase to maintain $\mathrm{Rb}$ in dephosphorylated form (70).
Unlike Cer, few studies have addressed the roles of SK and $\mathrm{S} 1 \mathrm{P}$ in senescence. One recent study suggested that hTERT is a direct target of S1P (28). In human lung cancer cells, SK2 knockdown or pharmacological inhibition decreased hTERT expression at the protein level, suggesting that SK2-generated S1P promotes hTERT stability. Mouse embryonic fibroblasts (MEFs) lacking SK2 became senescent at passage 5 , whereas senescence appeared in WT or SK1-deficient MEFs after seven passages.

On the other hand, pharmacological inhibition of SK1, SK2, or Des1 induced an increase of $\mathrm{p} 53$ and $\mathrm{p} 21$ expression in prostate cancer cells that was associated with decreased cell proliferation (71), a mechanism mediated by ROS. Interestingly, silencing either SK1 or Des1 expression with siRNA did not have any effect, but combined silencing increased p21 expression. This suggests a role for dhCer in inducing growth arrest in cancer cells and a synergistic effect of Des1 and SK1 knockdown on the development of senescence.

Similar to the results in SK2-deficient MEFs, CERT-deficient MEFs showed decreased proliferation, stopped dividing after six passages, and exhibited $G_{1}$ arrest, increased p16 expression, and decreased total SM and Cer, whereas total hexosylceramide (HexCer) increased. Functionally, CERT deficiency induced ER stress, altered Golgi dynamics, and dysregulated mitochondria, leading to mitophagy and senescence (72).

\section{Role of sphingolipids in senescence and age-related diseases}

The roles of senescence have been studied in many pathologies, such as cancer and metabolic and cardiovascular diseases, and in different animal models. Interestingly, sphingolipids and mechanisms upstream and downstream of sphingolipid metabolism are implicated in regulating senescence in the context of those pathologies (Table 2).

Cancer. Senescence provides a mechanism of cancer prevention by countering tumor growth. An exhaustive literature on the role of sphingolipids in cancer has established some links 
with senescence regulation, especially in liver cancer (73). Tumor suppressors and oncogenes affect sphingolipid metabolism, modifying the balance between pro-senescence and anti-senescence sphingolipids. Similarly, sphingolipids have a role in the response to cancer therapy through their regulation of senescence.

In general, induction of senescence by oncogenes such as Ras is p53-dependent, but maintaining the senescent state depends on $\mathrm{p} 16$ and $\mathrm{p} 21$. The protein phosphatase PP1CA, a direct target of Cer, was shown to regulate oncogene-induced senescence. Loss of PP1CA function bypassed p53-induced growth arrest (74). Also, PP1CA shRNA impaired p53's ability to induce p21 and blocked $\mathrm{pRb}$ dephosphorylation. PP1CA downregulation increased the cells' ability to form colonies, whereas PP1CA overexpression reduced colony formation. Therefore, PP1CA acts as a tumor suppressor through its contribution to Ras-induced senescence, raising the possibility that Cer, through PP1CA activation, may be a key signaling molecule in oncogene-induced senescence.

In vitro studies suggest that the p53's tumor-suppressive effects could be mediated, at least in part, through the downregulation of SK1, leading to decreased S1P levels and increased Cer and Sph levels, which then mediate apoptosis and senescence. In other words, p53 may act upstream of sphingolipid metabolism to induce its tumor suppressor effects. In line with this, it was shown that DNA-damaging agents induce p53-dependent proteolysis of SK1 in several cell types (75). In p53-KO mice, which develop thymic lymphomas, SK1 expression and S1P levels were higher, whereas Cer levels were decreased in thymus, compared with WT (76). Remarkably, p53-deficient mice lacking SK1 (double KO) were almost completely protected from thymic lymphoma, as shown by either absence or marked decrease of thymic mass. Thymus from SK1-deficient mice showed less S1P and more Cer than that from control. Sph levels were indeed very high in the double-KO thymus. There was no change in apoptosis, but there was a substantial increase of p21 and SA $\beta$-gal, indicating induction of senescence, which was postulated as the mechanism preventing tumor development in the p53/SK1 double-KO mice.

In additional studies, crossing cancer-prone p53 heterozygote mice with SK1-KO mice protected them from developing lymphoma, osteosarcoma, lung adenocarcinoma, and rhabdomyosarcoma. Restoring SK1 downregulation in p53 heterozygote mice normalized Cer levels and expression of the cell cycle inhibitors p16 and p21 (76).

Additional studies have examined targeting the SK2 isoform as a possible method to induce senescence in cancer cells and tumor suppression. Knockdown of SK2 in lung carcinoma cells before implantation into mice completely abolished tumor growth (28). An SK inhibitor induced tumor suppression in a xenograft model of lung carcinoma in immunodeficient SCID mice. hTERT expression gave resistance to SK inhibitor-induced tumor suppression and increased tumor volume. These results suggest that inhibition of S1P-hTERT binding in cancer cells may induce senescence and suppress tumor growth.

Therapy-induced senescence occurs in cancer, and noncancer cells after exposure to chemotherapy or radiotherapy, and sphingolipids, especially Cer, are implicated in this response. For example, gemcitabine does not completely kill AsPC-1 and PANC-1 human pancreatic cancer cell lines in vitro (77). Even at high concentration, $30 \%-40 \%$ of cells remained viable and became senescent. Adding exogenous SM enhanced chemosensitivity through an increase in Cer, induced apoptosis up to $90 \%$, and decreased cell cycle arrest and senescence. The degree of change in Cer levels determined the cell response, such that exogenous C8-Cer did not inhibit cell cycle progression at low concentration, induced senescence at moderate concentration, and induced apoptosis at high concentration (77).

In cancer therapy, senescence can lead to inflammation, secondary tumors, or cancer relapse (78). In this context, therapeutic strategies that increase Cer levels and decrease S1P levels could very efficiently induce senescence and prevent carcinogenesis.

Although senescence is considered a tumor suppressor mechanism, a few studies suggest that, in prostate cancer (79) and colorectal cancer (80), senescence promotes tumor growth and chemoresistance mostly through protumorigenic effects of SASP. A complex dialogue between tumor senescent cells, tumor nonsenescent cells, and nontumor cells regulates the outcome of carcinogenesis and response to cancer therapy. Further investigation is needed to better understand this interplay and the role of senescence and sphingolipids in the context of cancer.

Fibrosis and organ aging. Accumulating evidence suggests that senescence exerts antifibrotic effects in different organs such as skin, liver, kidney, and heart. Fibrosis is also increased in many organs throughout life. Sphingolipids are involved in the response to acute injury and wound healing, but few studies have connected sphingolipid metabolism and the regulation of senescence in the context of different organ fibrosis. Here we present some promising parallels between sphingolipids, senescence, and fibrosis.

In a mouse model of cutaneous wound healing, the conversion of fibroblast phenotype from activated to senescent was a key event, decreasing tissue fibrosis through an ROS- and p38-dependent mechanism and leading to 16 induction (81). With increasing age, nSMase and CerS activities are reduced in the whole epidermis (82), and this could explain decreased Cer content observed in the skin during aging, which is associated with an increase of fibrosis.

In a mouse model of renal fibrosis induced by unilateral ureteral obstruction, p16-deficient mice showed more tubular and interstitial cell proliferation, more matrix deposition, and lack of senescent cells (83). Thus, in this model, p16-mediated senescence controls cell proliferation and limits matrix deposition and fibrosis after injury. On the other hand, in a model of ischemia/ reperfusion injury, loss of p16 reduced injury-induced fibrosis, suggesting that in this case senescent cells contributed to the fibrosis (84). During aging, nSMase and CerS activities increase, and HexCer and lactosylceramide (LacCer) accumulate in mouse kidney (85). Caloric restriction reduced those activities, reduced levels of sphingolipids, and attenuated the aging processes. Therefore, this study suggested that HexCer and LacCer could be important mediators of aging in the kidney (85).

Senescence was shown to limit cardiac fibrosis after myocardial infarction (MI) (86), whereby p53 deficiency decreases the accumulation of senescent fibroblasts and increases matrix deposition after MI. In mice, Cer accumulates in the heart during aging and is associated with cardiac hypertrophy (87).

Carbon tetrachloride-induced ( $\mathrm{CCl}_{4}$-induced) liver fibrosis results in accumulation of senescent hepatic stellate cells in the fibrotic liver (88). p53-deficient mice show more fibrotic tissue containing more proliferating cells and fewer senescent cells compared 
with controls. This response is enhanced in p53/p16 double-KO mice. In rat liver, there is an increase of aSMase, nSMase, aCDase, and nCDase activities (89). Indeed, in old rat livers, NikolovaKarakashian and coworkers showed increases in activities of several sphingolipid enzymes (especially nSMase) and increased Cer and Sph levels, and these changes correlated with increased inflammatory responses (90-92). Moreover, SMS and CerS activities were lower in old livers compared with young ones. Additionally, a recent study connected aCDase and Cer to liver fibrosis (93), but no direct connection has been made between Cer induction and the liver during aging, or potential effects on liver function.

Type 2 diabetes. Significant evidence points to roles of sphingolipids in senescence and insulin resistance. Mice fed with a high-fat diet (HFD) develop type 2 diabetes, and the pathology positively correlates with senescence as evaluated by increased SA $\beta$-gal and decreased $\beta$ cell proliferation (94). Accumulating evidence increasingly implicates sphingolipids, especially Cer, in senescence and insulin resistance. Levels of sphingolipids in adipose tissue, liver, and skeletal muscle change significantly during aging. Adipocytes from old mice have higher levels of Cer, SM, GluCer, and C1P compared with adipocytes from young mice (95), but no changes in Sph and S1P were observed during aging. De novo Cer synthesis is involved in the increase of IL- 6 and TNF- $\alpha$ production, and mRNA levels of these cytokines are higher in adipocytes from old mice. Young adipocytes treated with Cer secrete the same levels of cytokines as old adipocytes, suggesting a role for the increase in Cer in driving the inflammatory response. Moreover, HFD increases CerS6 expression and C16-Cer levels in adipose tissue. Indeed, CerS6-deficient mice have less C16-Cer and are protected from HFD-induced obesity and glucose intolerance (96). CerS6 deficiency also decreases inflammation in adipose tissue, as shown by decreased macrophage infiltration and decreased expression of proinflammatory genes (96). Confirming preclinical results, a positive correlation has been established between CerS6 mRNA in adipose tissue and insulin resistance in obese subjects (96).

Furthermore, C2-Cer induces insulin resistance in hepatocytes of young rats, and treatment of insulin-resistant young hepatocytes or old hepatocytes with Myr, which inhibits de novo synthesis of Cer and all sphingolipids, increases insulin sensitivity (97). In mouse C2C12 myoblasts, C2-Cer promotes senescence as revealed by increased SA $\beta$-gal, p53, and p 21 expression, decreased BrdU incorporation, and induction of $\mathrm{G}_{2} / \mathrm{M}$ cell cycle arrest. C2-Cer also inhibits insulin signaling (98).

Thus, Cer plays a central role in the regulation of senescence, insulin sensitivity, and inflammation in adipose tissue and other tissues during aging.

Atherosclerosis. With age, alterations in sphingolipids in the heart and vessels accompany altered cardiovascular function. Studies show effects of sphingolipids on vascular cell biology, especially on the induction of endothelial cell senescence, and increasing evidence points to sphingolipid roles in this pathology and to possible relationships with senescence. Indeed, it should be noted that blood levels of specific Cers are emerging as clinical markers of atherosclerotic diseases (99).

Evaluation of sphingolipid levels in mesenteric small arteries from sheep during aging shows that old animals have increases in long-chain Cer, C14-C20 but mostly C16, and no change in levels of very-long-chain Cer, Sph, or S1P. aSMase and nSMase activities increase with age, which is most likely the cause of elevated Cer (100). In aged endothelia, nSMase activity is high, Cer is increased, and PP2A activity (target of Cer) is also increased. Mechanistically, these changes lead to dysregulation of endothelial NOS phosphorylation and inactivation, which are associated with age-induced loss of vasomotor function (101). C6-Cer induces senescence in HUVECs, characterized as cell enlargement, cell cycle arrest in $G_{1}$ phase, and expression of SA $\beta$-gal (102). Ex vivo experiments using vessel rings showed that GW4869, an nSMase inhibitor, prevents age-dependent loss of vasomotor function and improves whole-vessel vasocompliance (103). Another study confirmed these results (104), supporting the deleterious effects of nSMase and Cer on vascular tissue during aging.

Endothelial senescence is associated with aging and cardiovascular diseases (105) and can be modulated by the level of cytokines such as IL-1 (106). Aging impairs endothelial functions including chemotaxis, wound healing, and morphogenesis. In atherosclerotic lesions of mouse aorta, endothelial cells are senescent and S1PR2 expression is dramatically increased (107). In the same way, in vitro, young endothelial cells express low levels of S1PR2, whereas this receptor is highly expressed in senescent endothelial cells. Overexpression of S1PR2 in young cells induced senescence-associated endothelial impairments. In contrast, inhibition of S1PR2 signaling in senescent cells decreased senescence and restored endothelial function (107). Thus, S1PR2 appears to play a critical role in promoting endothelial senescence. From a therapeutic perspective, S1PR2 appears to be a candidate target to prevent vascular aging. Inhibiting S1PR2 signaling with an antagonist or inhibitor might be a way to prevent age-related impairment of endothelial functions.

In more advanced atherosclerotic lesions, senescence could worsen pathology and trigger complications. MMPs released by senescent vascular cells can degrade the fibrous cap and make the atheroma less stable, leading to stroke or MI (108). S1P and its receptor S1PR1 have been shown to have vasculoprotective effects. It would be interesting to study whether some of these effects are related to a potential inhibition of endothelial senescence.

Immunosenescence. Some studies have also addressed the roles of sphingolipids in immune and adaptive cell senescence. The levels of most sphingolipids are higher in $\mathrm{CD} 4^{+} \mathrm{T}$ cells from aged mice compared with young ones, and addition of exogenous C2- and C6-Cer inhibited CD4 ${ }^{+} \mathrm{T}$ cell proliferation (109). This suggests that age-related Cer increases might contribute to the proliferative defects observed in aged $\mathrm{CD} 4^{+} \mathrm{T}$ cells. Peritoneal macrophages isolated from old mice showed an increase of $\mathrm{NF}-\kappa \mathrm{B}$ activation compared with those from young mice. Exogenous Cer induced $\mathrm{NF}-\kappa \mathrm{B}$ activation and COX-2 expression in young macrophages (110), showing that aging and Cer have the same effect on macrophages. The link is not yet fully established, but Cer could be responsible for the dysregulation of innate and adaptive immunity observed during aging.

Other pathologies. Senescence has been associated with many other diseases, among them, Alzheimer's disease (AD) (111), Parkinson's disease (112), aneurysms (113), osteoarthritis (114), glaucoma (115), macular degeneration (116), chronic obstructive pulmonary disease (117), and age-related sarcopenia. 
$\mathrm{AD}$ is of particular interest, as significant literature links sphingolipids to AD. C6-Cer promotes amyloid $\beta$-peptide (A $\beta$ ) biogenesis in a human neuroglioma cell line by stabilizing $\beta$-secretase, leading to an increase in the cleavage of the amyloid precursor protein (118). aSMase activity is increased in the brains of AD mice, and partial inhibition of aSMase in a mouse model of familial AD decreased AD-like symptoms such as $A \beta$ deposition and memory impairment through the restoration of lysosomal biogenesis (119). nSMase activity is also higher with age in striatum, hippocampus, and frontal cortex. This correlates with increased expression of inflammatory markers such as IL-1 $\beta$ (120). A recent study showed that nSMase2-deficient mice have less severe AD-like symptoms than control mice (121). The underlying mechanism was a reduction of brain exosomes in the nSMase2-deficient mice. Indeed, this study also showed that Cer-enriched exosomes aggravate $\mathrm{AD}$ pathology. Thus, as discussed in the section below, Cer could play a major role in $\mathrm{AD}$ pathogenesis through the induction of senescence and inflammation.

\section{Clinical data}

A flurry of recent studies have addressed the potential roles of blood sphingolipids as possible biomarkers for aging and agingrelated diseases. Clinical studies have made significant correlations between the levels of sphingolipids, specifically Cer, and sphingolipid enzymes and different pathologies such as atherosclerosis, cataract, AD, and type 2 diabetes (96, 122-124).

Interestingly, centenarians, considered a human model of healthy aging, exhibit increased SM and very-long-chain Cer in serum compared with younger elderly subjects (125). Another study showed increased plasma levels of some long-chain Cer in centenarians compared with aged subjects, but other long-chain Cer and GluCer are decreased (126).

Plasma SM and Cer levels correlate with increased risk of coronary heart disease $(127,128)$. A study suggested that reducing Cer levels in the heart might improve ventricular function (129). Patients with chronic heart failure exhibit a positive correlation between plasma Cer levels and heart failure severity (122).

In human lens, Cer and dhCer levels increase with age (123) and are barely detectable before the age of 30. Modification of the lipid composition may impact physical properties of the fiber cell membranes and may be associated with cataract formation (130).
SM and Cer may be relevant biomarkers and therapeutic targets in $\mathrm{AD}$. Cer content is elevated in the cerebrospinal fluid of $\mathrm{AD}$ patients (131). Increases of C24:0 Cer were observed in vulnerable brain regions of $\mathrm{AD}$ patients such as the middle frontal gyrus, and these changes were associated with oxidative stress (132). SM levels are associated with AD severity in the brain and the blood of patients (133). Higher baseline serum levels of Cer were associated with an increased risk of AD (124).

Thus, specific sphingolipids and specific Cers are emerging as possible diagnostics, biomarkers, and indices of several human diseases, a process increasingly enabled by the evolving mass spectrometry technology directed at measuring sphingolipids.

\section{Conclusions}

Significant and wide-ranging evidence defines critical roles of sphingolipid enzymes and pathways in aging of model organisms. Likewise, increasing evidence points to multiple roles of several bioactive sphingolipids, especially Cer and S1P, in mechanisms of mammalian cell and tissue senescence. Further investigations are needed to better understand the specific mechanisms connecting bioactive sphingolipids to specific aspects of the senescence program, including regulation by stress stimuli and p53, and participation in growth arrest, SASP, and other aspects of the senescence response. Beyond that, there is a need to understand the roles of sphingolipids in the overall aging process and in age-related diseases.

\section{Acknowledgments}

This work was supported by a Veterans Affairs Merit Award and NIH grants GM097741 and P01 CA097132 to LMO and R35 GM118128 to YAH, as well as a postdoctoral fellowship (SPE20140129283) from the French Medical Research Foundation (FRM) to MT.

Address correspondence to: Lina M. Obeid, Department of Medicine, Stony Brook University, Health Science Center, L-4, 179, Stony Brook, New York 11794-8430, USA. Phone: 631.444.2641; Email: lina.obeid@stonybrookmedicine.edu. Or to: Yusuf A. Hannun, Stony Brook University, Health Science Center, L-4, 182, Stony Brook, New York 11794-8430, USA. Phone: 631.444.8067; Email:yusuf.hannun@stonybrookmedicine.edu.
1. Goñi FM, Alonso A. Biophysics of sphingolipids I. Membrane properties of sphingosine, ceramides and other simple sphingolipids. Biochim Biophys Acta. 2006;1758(12):1902-1921.

2. Rietveld A, Simons K. The differential miscibility of lipids as the basis for the formation of functional membrane rafts. Biochim Biophys Acta. 1998;1376(3):467-479.

3. Hannun YA, Obeid LM. Principles of bioactive lipid signalling: lessons from sphingolipids. Nat Rev Mol Cell Biol. 2008;9(2):139-150.

4. Hannun YA, Obeid LM. Sphingolipids and their metabolism in physiology and disease. Nat Rev Mol Cell Biol. 2018;19(3):175-191.

5 . Flatt T. A new definition of aging? Front Genet. 2012;3:148.

6. Singh P, Li R. Emerging roles for sphingolipids in cellular aging [published online ahead of print
December 19, 2017]. Curr Genet. https://doi. org/10.1007/s00294-017-0799-z.

7. Muñoz-Espín D, Serrano M. Cellular senescence: from physiology to pathology. Nat Rev Mol Cell Biol. 2014;15(7):482-496.

8. Hayflick L, Moorhead PS. The serial cultivation of human diploid cell strains. Exp Cell Res. 1961;25:585-621.

9. Lopez-Otin C, Blasco MA, Partridge L, Serrano M, Kroemer G. The hallmarks of aging. Cell. 2013;153(6):1194-1217.

10. Niccoli T, Partridge L. Ageing as a risk factor for disease. Curr Biol. 2012;22(17):R741-R752.

11. Hannun YA, Obeid LM. Many ceramides. J Biol Chem. 2011;286(32):27855-27862.

12. Hernandez-Corbacho MJ, Salama MF, Canals D, Senkal CE, Obeid LM. Sphingolipids in mitochondria. Biochim Biophys Acta. 2017;1862(1):56-68.
13. Merrill AH. De novo sphingolipid biosynthesis: a necessary, but dangerous, pathway. J Biol Chem. 2002;277(29):25843-25846.

14. Hornemann T, Wei Y, von Eckardstein A. Is the mammalian serine palmitoyltransferase a high-molecular-mass complex? Biochem J. 2007;405(1):157-164.

15. Hornemann T, et al. The SPTLC3 subunit of serine palmitoyltransferase generates short chain sphingoid bases. J Biol Chem. 2009;284(39):26322-26330.

16. Mullen TD, Hannun YA, Obeid LM. Ceramide synthases at the centre of sphingolipid metabolism and biology. Biochem J. 2012;441(3):789-802.

17. Milhas D, Clarke CJ, Hannun YA. Sphingomyelin metabolism at the plasma membrane: implications for bioactive sphingolipids. FEBS Lett. 2010;584(9):1887-1894. 
18. Adada M, Luberto C, Canals D. Inhibitors of the sphingomyelin cycle: sphingomyelin synthases and sphingomyelinases. Chem Phys Lipids. 2016;197:45-59.

19. Kitatani K, Idkowiak-Baldys J, Hannun YA. The sphingolipid salvage pathway in ceramide metabolism and signaling. Cell Signal. 2008;20(6):1010-1018.

20. Ogretmen B, et al. Biochemical mechanisms of the generation of endogenous long chain ceramide in response to exogenous short chain ceramide in the A549 human lung adenocarcinoma cell line. Role for endogenous ceramide in mediating the action of exogenous ceramide. JBiol Chem. 2002;277(15):12960-12969.

21. Mao C, Obeid LM. Ceramidases: regulators of cellular responses mediated by ceramide, sphingosine, and sphingosine-1-phosphate. Biochim Biophys Acta . 2008;1781(9):424-434.

22. Maceyka M, et al. SphK1 and SphK2, sphingosine kinase isoenzymes with opposing functions in sphingolipid metabolism. J Biol Chem. 2005;280(44):37118-37129.

23. Hait NC, et al. Regulation of histone acetylation in the nucleus by sphingosine-1-phosphate. Science. 2009;325(5945):1254-1257.

24. Sanchez T, Hla T. Structural and functional characteristics of S1P receptors. J Cell Biochem. 2004;92(5):913-922.

25. Gault CR, Obeid LM, Hannun YA. An overview of sphingolipid metabolism: from synthesis to breakdown. Adv Exp Med Biol. 2010;688:1-23.

26. Strub GM, Maceyka M, Hait NC, Milstien S, Spiegel S. Extracellular and intracellular actions of sphingosine-1-phosphate. Adv Exp Med Biol. 2010;688:141-155

27. Strub GM, et al. Sphingosine-1-phosphate produced by sphingosine kinase 2 in mitochondria interacts with prohibitin 2 to regulate complex IV assembly and respiration. FASEB J. 2011;25(2):600-612.

28. Panneer Selvam S, et al. Binding of the sphingolipid S1P to hTERT stabilizes telomerase at the nuclear periphery by allosterically mimicking protein phosphorylation. Sci Signal. 2015;8(381):ra58.

29. Mitra P, Oskeritzian CA, Payne SG, Beaven MA, Milstien S, Spiegel S. Role of ABCC1 in export of sphingosine-1-phosphate from mast cells. Proc Natl Acad Sci U S A. 2006;103(44):16394-16399.

30. Nagahashi M, et al. Sphingosine-1-phosphate transporters as targets for cancer therapy. Biomed Res Int. 2014;2014:651727.

31. Vu TM, et al. Mfsd2b is essential for the sphingosine-1-phosphate export in erythrocytes and platelets. Nature. 2017;550(7677):524-528.

32. Taha TA, Argraves KM, Obeid LM. Sphingosine-1-phosphate receptors: receptor specificity versus functional redundancy. Biochim Biophys Acta. 2004;1682(1-3):48-55.

33. Proia RL, Hla T. Emerging biology of sphingosine-1-phosphate: its role in pathogenesis and therapy. JClin Invest. 2015;125(4):1379-1387.

34. Pulkoski-Gross MJ, Donaldson JC, Obeid LM. Sphingosine-1-phosphate metabolism: a structural perspective. Crit Rev Biochem Mol Biol. 2015;50(4):298-313.

35. Mullershausen F, Zecri F, Cetin C, Billich A,
Guerini D, Seuwen K. Persistent signaling induced by FTY720-phosphate is mediated by internalized S1P1 receptors. Nat Chem Biol. 2009;5(6):428-434.

36. Willis MA, Cohen JA. Fingolimod therapy for multiple sclerosis. Semin Neurol. 2013;33(1):37-44.

37. Schnute ME, et al. Modulation of cellular S1P levels with a novel, potent and specific inhibitor of sphingosine kinase-1. Biochem J. 2012;444(1):79-88.

38. French KJ, Upson JJ, Keller SN, Zhuang Y, Yun JK, Smith CD. Antitumor activity of sphingosine kinase inhibitors. JPharmacol Exp Ther. 2006;318(2):596-603.

39. French KJ, et al. Pharmacology and antitumor activity of ABC294640, a selective inhibitor of sphingosine kinase-2. J Pharmacol Exp Ther. 2010;333(1):129-139.

40. Chalfant CE, Szulc Z, Roddy P, Bielawska A, Hannun YA. The structural requirements for ceramide activation of serine-threonine protein phosphatases. J Lipid Res. 2004;45(3):496-506.

41. Wlodarchak N, Xing Y. PP2A as a master regulator of the cell cycle. Crit Rev Biochem Mol Biol. 2016;51(3):162-184.

42. Yamaji T, Hanada K. Sphingolipid metabolism and interorganellar transport: localization of sphingolipid enzymes and lipid transfer proteins. Traffic. 2015;16(2):101-122.

43. Jiang Y, et al. Combinatorial therapies improve the therapeutic efficacy of nanoliposomal ceramide for pancreatic cancer. Cancer Biol Ther. 2011;12(7):574-585.

44. Novgorodov SA, Gudz TI, Obeid LM. Long-chain ceramide is a potent inhibitor of the mitochondrial permeability transition pore. J Biol Chem. 2008;283(36):24707-24717.

45. de Magalhães JP, Passos JF. Stress, cell senescence and organismal ageing. Mech Ageing Dev. 2018;170:2-9.

46. Childs BG, Durik M, Baker DJ, van Deursen JM. Cellular senescence in aging and age-related disease: from mechanisms to therapy. Nat Med. 2015;21(12):1424-1435.

47. Childs BG, Baker DJ, Kirkland JL, Campisi J, van Deursen JM. Senescence and apoptosis: dueling or complementary cell fates? EMBO Rep. 2014;15(11):1139-1153

48. Soto-Gamez A, Demaria M. Therapeutic interventions for aging: the case of cellular senescence. Drug Discov Today. 2017;22(5):786-795.

49. van Deursen JM. The role of senescent cells in ageing. Nature. 2014;509(7501):439-446.

50. Rossiello F, Herbig U, Longhese MP, Fumagalli M, d'Adda di Fagagna F. Irreparable telomeric DNA damage and persistent DDR signalling as a shared causative mechanism of cellular senescence and ageing. Curr Opin Genet Dev. 2014;26:89-95.

51. Guo X, et al. Dysfunctional telomeres activate an ATM-ATR-dependent DNA damage response to suppress tumorigenesis. EMBOJ. 2007;26(22):4709-4719.

52. Campisi J, d'Adda di Fagagna F. Cellular senescence: when bad things happen to good cells. Nat Rev Mol Cell Biol. 2007;8(9):729-740.

53. Krishnamurthy J, et al. Ink4a/Arf expression is a biomarker of aging. J Clin Invest. 2004;114(9):1299-1307.

54. Gil J, Peters G. Regulation of the INK4b-ARF-
INK4a tumour suppressor locus: all for one or one for all. Nat Rev Mol Cell Biol. 2006;7(9):667-677.

55. Johmura Y, et al. Necessary and sufficient role for a mitosis skip in senescence induction. Mol Cell. 2014;55(1):73-84.

56. Gorgoulis VG, Halazonetis TD. Oncogene-induced senescence: the bright and dark side of the response. Curr Opin Cell Biol. 2010;22(6):816-827.

57. Di Micco R, et al. Oncogene-induced senescence is a DNA damage response triggered by DNA hyper-replication. Nature. 2006;444(7119):638-642.

58. Macip S, et al. Inhibition of p21-mediated ROS accumulation can rescue $\mathrm{p} 21$-induced senes-

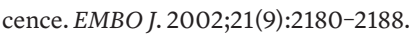

59. Barascu A, et al. Oxidative stress induces an ATM-independent senescence pathway through p38 MAPK-mediated lamin B1 accumulation. EMBO J. 2012;31(5):1080-1094.

60. Dimri GP, et al. A biomarker that identifies senescent human cells in culture and in aging skin in vivo. Proc Natl Acad Sci US A. 1995;92(20):9363-9367.

61. Freund A, Laberge RM, Demaria M, Campisi J Lamin B1 loss is a senescence-associated biomarker. Mol Biol Cell. 2012;23(11):2066-2075.

62. Coppé JP, et al. Senescence-associated secretory phenotypes reveal cell-nonautonomous functions of oncogenic RAS and the p53 tumor suppressor. PLoS Biol. 2008;6(12):2853-2868.

63. Salminen A, Kauppinen A, Kaarniranta K. Emerging role of NF- $\mathrm{BB}$ signaling in the induction of senescence-associated secretory phenotype (SASP). Cell Signal. 2012;24(4):835-845.

64. Venable ME, Lee JY, Smyth MJ, Bielawska A, Obeid LM. Role of ceramide in cellular senescence. J Biol Chem. 1995;270(51):30701-30708.

65. Meacci E, Vasta V, Neri S, Farnararo M, Bruni P. Activation of phospholipase D in human fibroblasts by ceramide and sphingosine: evaluation of their modulatory role in bradykinin stimulation of phospholipase D. Biochem Biophys Res Commun. 1996;225(2):392-399.

66. Dbaibo GS, et al. p53-dependent ceramide response to genotoxic stress. JClin Invest. 1998;102(2):329-339.

67. Lee JY, Leonhardt LG, Obeid LM. Cell-cycledependent changes in ceramide levels preceding retinoblastoma protein dephosphorylation in G2/M. Biochem J. 1998;334(pt 2):457-461.

68. Mouton RE, Venable ME. Ceramide induces expression of the senescence histochemica marker, beta-galactosidase, in human fibroblasts. Mech Ageing Dev. 2000;113(3):169-181.

69. Lee JY, Bielawska AE, Obeid LM. Regulation of cyclin-dependent kinase 2 activity by ceramide. Exp Cell Res. 2000;261(2):303-311.

70. Chao R, Khan W, Hannun YA. Retinoblastoma protein dephosphorylation induced by D-erythro-sphingosine. J Biol Chem 1992;267(33):23459-23462.

71. McNaughton M, Pitman M, Pitson SM, Pyne NJ, Pyne S. Proteasomal degradation of sphingosine kinase 1 and inhibition of dihydroceramide desaturase by the sphingosine kinase inhibitors, SKi or ABC294640, induces growth arrest in androgen-independent LNCaP-AI prostate cancer cells. Oncotarget. 2016;7(13):16663-16675.

72. Rao RP, et al. Ceramide transfer protein deficiency compromises organelle function and 
leads to senescence in primary cells. PLoS One. 2014;9(3):e92142.

73. Aravinthan AD, Alexander GJM. Senescence in chronic liver disease: is the future in aging? JHepatol. 2016;65(4):825-834.

74. Castro ME, et al. PPP1CA contributes to the senescence program induced by oncogenic Ras. Carcinogenesis. 2008;29(3):491-499.

75. Taha TA, et al. Down-regulation of sphingosine kinase- 1 by DNA damage: dependence on proteases and p53.JBiol Chem. 2004;279(19):20546-20554.

76. Heffernan-Stroud LA, Helke KL, Jenkins RW, De Costa AM, Hannun YA, Obeid LM. Defining a role for sphingosine kinase 1 in p53-dependent tumors. Oncogene. 2012;31(9):1166-1175.

77. Modrak DE, Leon E, Goldenberg DM, Gold DV. Ceramide regulates gemcitabine-induced senescence and apoptosis in human pancreatic cancer cell lines. Mol Cancer Res. 2009;7(6):890-896.

78. Schosserer M, Grillari J, Breitenbach M. The dual role of cellular senescence in developing tumors and their response to cancer therapy. Front Oncol. 2017;7:278.

79. Toso A, et al. Enhancing chemotherapy efficacy in Pten-deficient prostate tumors by activating the senescence-associated antitumor immunity. Cell Rep. 2014;9(1):75-89.

80. Pribluda A, et al. A senescence-inflammatory switch from cancer-inhibitory to cancer-promoting mechanism. Cancer Cell. 2013;24(2):242-256.

81. Jun JI, Lau LF. The matricellular protein CCN1 induces fibroblast senescence and restricts fibrosis in cutaneous wound healing. Nat Cell Biol. 2010;12(7):676-685.

82. Jensen JM, et al. Acid and neutral sphingomyelinase, ceramide synthase, and acid ceramidase activities in cutaneous aging. Exp Dermatol. 2005;14(8):609-618.

83. Wolstein JM, Lee DH, Michaud J, Buot V, Stefanchik B, Plotkin MD. INK4a knockout mice exhibit increased fibrosis under normal conditions and in response to unilateral ureteral obstruction. Am J Physiol Renal Physiol. 2010;299(6):F1486-F1495.

84. Braun $\mathrm{H}$, et al. Cellular senescence limits regenerative capacity and allograft survival. J Am Soc Nephrol. 2012;23(9):1467-1473.

85. Hernández-Corbacho MJ, et al. Accumulation of long-chain glycosphingolipids during aging is prevented by caloric restriction. PLoS One. 2011;6(6):e20411.

86. Zhu F, et al. Senescent cardiac fibroblast is critical for cardiac fibrosis after myocardial infarction. PLoS One. 2013;8(9):e74535.

87. Rodríguez-Calvo R, et al. Peroxisome proliferator-activated receptor alpha down-regulation is associated with enhanced ceramide levels in age-associated cardiac hypertrophy. J Gerontol A Biol Sci Med Sci. 2007;62(12):1326-1336.

88. Krizhanovsky V, et al. Senescence of activated stellate cells limits liver fibrosis. Cell. 2008;134(4):657-667.

89. Sacket SJ, Chung HY, Okajima F, Im DS. Increase in sphingolipid catabolic enzyme activity during aging. Acta Pharmacol Sin. 2009;30(10):1454-1461.

90. Lightle SA, Oakley JI, Nikolova-Karakashian MN. Activation of sphingolipid turnover and chronic generation of ceramide and sphingosine in liver during aging. Mech Ageing Dev. 2000;120(1-3):111-125.

91. Rutkute K, Karakashian AA, Giltiay NV, Dobierzewska A, Nikolova-Karakashian MN. Aging in rat causes hepatic hyperresposiveness to interleukin-1beta which is mediated by neutral sphingomyelinase-2. Hepatology. 2007;46(4):1166-1176.

92. Nikolova-Karakashian M, Karakashian A, Rutkute K. Role of neutral sphingomyelinases in aging and inflammation. Subcell Biochem. 2008;49:469-486.

93. Chen JY, et al. Tricyclic antidepressants promote ceramide accumulation to regulate collagen production in human hepatic stellate cells. Sci Rep. 2017;7:44867.

94. Sone H, Kagawa Y. Pancreatic beta cell senescence contributes to the pathogenesis of type 2 diabetes in high-fat diet-induced diabetic mice. Diabetologia. 2005;48(1):58-67.

95. Wu D, et al. Aging up-regulates expression of inflammatory mediators in mouse adipose tissue. JImmunol. 2007;179(7):4829-4839.

96. Turpin SM, et al. Obesity-induced Cers6dependent $\mathrm{C} 16: 0$ ceramide production promotes weight gain and glucose intolerance. Cell Metab. 2014;20(4):678-686.

97. Babenko NA, Kharchenko VS. Ceramides inhibit phospholipase D-dependent insulin signaling in liver cells of old rats. Biochemistry Mosc. 2012;77(2):180-186.

98. Jadhav KS, Dungan CM, Williamson DL. Metformin limits ceramide-induced senescence in $\mathrm{C} 2 \mathrm{C} 12$ myoblasts. Mech Ageing Dev. 2013;134(11-12):548-559.

99. Iqbal J, Walsh MT, Hammad SM, Hussain MM. Sphingolipids and lipoproteins in health and metabolic disorders. Trends Endocrinol Metab. 2017;28(7):506-518

100. Ohanian J, Liao A, Forman SP, Ohanian V. Agerelated remodeling of small arteries is accompanied by increased sphingomyelinase activity and accumulation of long-chain ceramides. Physiol Rep. 2014;2(5):e12015.

101.Seals DR, Jablonski KL, Donato AJ. Aging and vascular endothelial function in humans. Clin Sci (Lond). 2011;120(9):357-375.

102.Gao YF, Chen X, Su XQ, Zhai ZH. [Rapid induction of senescence-like changes in human umbilic vein endothelial cells (HUVECs) by C6 ceramide] (article in Chinese). Shi Yan Sheng Wu Хие Bao. 2001;34(4):333-335.

103.Smith AR, Visioli F, Frei B, Hagen TM. Age-related changes in endothelial nitric oxide synthase phosphorylation and nitric oxide dependent vasodilation: evidence for a novel mechanism involving sphingomyelinase and ceramide-activated phosphatase 2A. Aging Cell. 2006;5(5):391-400.

104.Smith AR, Visioli F, Frei B, Hagen TM. Lipoic acid significantly restores, in rats, the agerelated decline in vasomotion. Br JPharmacol. 2008;153(8):1615-1622.

105. Zhao J, Garcia D, Gartung A, Lee MJ. Sphingosine-1-phosphate receptor subtype 2 signaling in endothelial senescence-associated functional impairments and inflammation. Curr Atheroscler Rep. 2015;17(5):504.

106. Maier JA, Voulalas P, Roeder D, Maciag T. Extension of the life-span of human endothelial cells by an interleukin-1 alpha antisense oligomer.
Science. 1990;249(4976):1570-1574.

107. Estrada R, et al. Up-regulating sphingosine 1-phosphate receptor-2 signaling impairs chemotactic, wound-healing, and morphogenetic responses in senescent endothelial cells. J Biol Chem. 2008;283(44):30363-30375.

108.Finn AV, Nakano M, Narula J, Kolodgie FD, Virmani R. Concept of vulnerable/unstable plaque. Arterioscler Thromb Vasc Biol. 2010;30(7):1282-1292.

109. Molano A, et al. Age-dependent changes in the sphingolipid composition of mouse $\mathrm{CD} 4^{+} \mathrm{T}$ cell membranes and immune synapses implicate glucosylceramides in age-related T cell dysfunction. PLoS One. 2012;7(10):e47650.

110.Wu D, Marko M, Claycombe K, Paulson KE, Meydani SN. Ceramide-induced and age-associated increase in macrophage COX-2 expression is mediated through up-regulation of NF-kappa B activity. J Biol Chem. 2003;278(13):10983-10992.

111. Bhat R, et al. Astrocyte senescence as a component of Alzheimer's disease. PLoS One. 2012;7(9):e45069.

112. Chinta SJ, Lieu CA, Demaria M, Laberge RM, Campisi J, Andersen JK. Environmental stress, ageing and glial cell senescence: a novel mechanistic link to Parkinson's disease? J Intern Med. 2013;273(5):429-436.

113. Fukazawa $\mathrm{R}$, et al. Coronary artery aneurysm induced by Kawasaki disease in children show features typical senescence. Circ J. 2007;71(5):709-715.

114. Price JS, et al. The role of chondrocyte senescence in osteoarthritis. Aging Cell. 2002;1(1):57-65.

115. Liton PB, Challa P, Stinnett S, Luna C, Epstein DL, Gonzalez P. Cellular senescence in the glaucomatous outflow pathway. Exp Gerontol. 2005;40(8-9):745-748.

116.Zhu D, Wu J, Spee C, Ryan SJ, Hinton DR. BMP4 mediates oxidative stress-induced retinal pigment epithelial cell senescence and is overexpressed in age-related macular degeneration. J Biol Chem. 2009;284(14):9529-9539.

117. Tsuji T, Aoshiba K, Nagai A. Alveolar cell senescence exacerbates pulmonary inflammation in patients with chronic obstructive pulmonary disease. Respiration. 2010;80(1):59-70.

118. Puglielli L, Ellis BC, Saunders AJ, Kovacs DM. Ceramide stabilizes beta-site amyloid precursor protein-cleaving enzyme 1 and promotes amyloid beta-peptide biogenesis. J Biol Chem. 2003;278(22):19777-19783

119. Lee JK, et al. Acid sphingomyelinase modulates the autophagic process by controlling lysosomal biogenesis in Alzheimer's disease. J Exp Med. 2014;211(8):1551-1570.

120. Crivello NA, Rosenberg IH, Dallal GE, Bielinski $\mathrm{D}$, Joseph JA. Age-related changes in neutral sphingomyelin-specific phospholipase $\mathrm{C}$ activity in striatum, hippocampus, and frontal cortex: implication for sensitivity to stress and inflammation. Neurochem Int. 2005;47(8):573-579.

121. Dinkins MB, et al. Neutral sphingomyelinase-2 deficiency ameliorates Alzheimer's disease pathology and improves cognition in the 5XFAD mouse. J Neurosci. 2016;36(33):8653-8667.

122.Yu J, et al. Ceramide is upregulated and associated with mortality in patients with chronic heart failure. Can JCardiol. 2015;31(3):357-363. 
123. Hughes JR, et al. Instability of the cellular lipidome with age. Age (Dordr). 2012;34(4):935-947.

124. Mielke MM, et al. Serum ceramides increase the risk of Alzheimer disease: the Women's Health and Aging Study II. Neurology. 2012;79(7):633-641.

125. Montoliu I, et al. Serum profiling of healthy aging identifies phospho- and sphingolipid species as markers of human longevity. Aging (Albany NY). 2014;6(1):9-25.

126. Jové $\mathrm{M}$, et al. A stress-resistant lipidomic signature confers extreme longevity to humans. J Gerontol A Biol Sci Med Sci. 2017;72(1):30-37.

127. Schissel SL, Tweedie-Hardman J, Rapp JH, Graham G, Williams KJ, Tabas I. Rabbit aorta and human atherosclerotic lesions hydrolyze the sphingomyelin of retained low-density lipoprotein. Proposed role for arterial-wall sphingomyelinase in subendothelial retention and aggregation of atherogenic lipoproteins. J Clin Invest. 1996;98(6):1455-1464.

128. Jiang XC, et al. Plasma sphingomyelin level as a risk factor for coronary artery disease. Arterioscler Thromb Vasc Biol. 2000;20 (12):2614-2618.

129. Chokshi A, et al. Ventricular assist device implan- tation corrects myocardial lipotoxicity, reverses insulin resistance, and normalizes cardiac metabolism in patients with advanced heart failure. Circulation. 2012;125(23):2844-2853.

130. Deeley JM, et al. Sphingolipid distribution changes with age in the human lens. J Lipid Res. 2010;51(9):2753-2760.

131. Satoi H, et al. Astroglial expression of ceramide in Alzheimer's disease brains: a role during neuronal apoptosis. Neuroscience. 2005;130(3):657-666.

132. Cutler RG, et al. Involvement of oxidative stress-induced abnormalities in ceramide and cholesterol metabolism in brain aging and Alzheimer's disease. Proc Natl Acad Sci U S A. 2004;101(7):2070-2075.

133. Varma VR, et al. Brain and blood metabolite signatures of pathology and progression in Alzheimer disease: a targeted metabolomics study. PLoS Med. 2018;15(1):e1002482.

134. D'Mello NP, Childress AM, Franklin DS, Kale SP, Pinswasdi C, Jazwinski SM. Cloning and characterization of LAG1, a longevity-assurance gene in yeast. J Biol Chem. 1994;269(22):15451-15459.

135. Huang X, Liu J, Dickson RC. Down-regulating sphingolipid synthesis increases yeast lifespan. PLoS Genet. 2012;8(2):e1002493.

136. Yi JK, et al. Aging-related elevation of sphingoid bases shortens yeast chronological life span by compromising mitochondrial function. Oncotarget. 2016;7(16):21124-21144.

137. Cutler RG, Thompson KW, Camandola S, Mack KT, Mattson MP. Sphingolipid metabolism regulates development and lifespan in Caenorhabditis elegans. Mech Ageing Dev. 2014;143-144:9-18.

138. Mosbech MB, et al. Functional loss of two ceramide synthases elicits autophagy-dependent lifespan extension in C. elegans. PLoS One. 2013;8(7):e70087.

139. Chan JP, et al. Loss of sphingosine kinase alters life history traits and locomotor function in Caenorhabditis elegans. Front Genet. 2017;8:132.

140. Yang Q, et al. Role of Drosophila alkaline ceramidase (Dacer) in Drosophila development and longevity. Cell Mol Life Sci. 2010;67(9):1477-1490.

141. Rao RP, et al. Ceramide transfer protein function is essential for normal oxidative stress response and lifespan. Proc Natl Acad Sci U S A. 2007;104(27):11364-11369. 